\title{
Geospatial-temporal Characteristics of Population Aging and Its Evolution Pattern in Henan
}

\author{
ZHANG Kaiguang $^{1, a^{*}}$, BA Mingting ${ }^{2, b}$ and MENG Hongling ${ }^{3, c}$ \\ ${ }^{1}$ Institute of 3S Technology, Zhengzhou Normal University, Zhengzhou 450044, China \\ azzgis@sina.com, bbmt1234@126.com, chnmhl@126.coml
}

\begin{abstract}
Keywords: population aging, aging level, aging rate, aging type, geospatial distribution,
\end{abstract} geospatial-temporal characteristics

\begin{abstract}
Population aging and aging rate is one of the world's most concerned population problems. In the paper, using geospatial statistical analysis methods, the geospatial-temporal characteristics and evolution pattern of aging level, causes, and increasing rate are studied in 2011-2015 in Henan province. The results show that the distribution of population aging levels has significant regional differences in the province, the aging level of southeastern part is significantly higher than that of other regions, The regions with low aging level have relatively developed economics, the economics development are relatively backward in the regions with high aging level and high population density. The main causes of aging is residual space type and low birth rate type. The aging rates have gradually been increasing, the differences between the cities have been enlarging, aging level and the aging rate has some negative correlation. The geospatial aggregation of aging levels is significant, southeast part is the high-high aggregation region and northern part is low-low aggregation region, but the aggregation characteristics are becoming weak. The aggregation characteristics of aging rate is relatively weak, there are some weak low-low aggregation characteristics around Zhengzhou, provincial capital, the geospatial distribution of aging rates is relatively balanced, the aging rate in central region is lower than the border region.
\end{abstract}

\section{Introduction}

Population aging and aging rate is one of the world's most concerned population problems. The high birth rate in the 20th century 50-70 years, and followed strict population control policy, make the population aging problem is becoming more and more seriously in recent China[1-4]. According to the goal of social development as 'promoting the balanced development of long-term population and building a population balanced society', currently it is a long-term and arduous task for China to ensure the limited population growth, make population adapt with economic and social development, control the aging level and rate, adjusting the age structure to reasonable range [5-7].

Aging population refers to the increasing process of the proportion of elderly population, by a certain age defined before, in the total population, which reflects the change of population age structure [8,9]. At present, the researches on population aging mainly concentrate on the variations about population aging spatial distribution, population aging growth and spatial migration patterns. In China population aging researches mainly concentrate on the economic relationship between population aging and the disappearance of the demographic dividend, at and above the provincial level, and get some valuable results [8-15].

Henan Province is located in the middle of China, on the middle and lower reaches of the Yellow River, in the second step to the third step of the transition zone, the land area of 167 thousand $\mathrm{km}^{2}$, accounting for $1.73 \%$ of the total area of the country, is China's most populated province. To 2015, the population reaches 106.62 million, is the most serious aging provinces with $9.4 \%$ aging level.

Based on the resident population data with different age levels, using geospatial statistical analysis methods, this paper studies the geospatial-temporal characteristics and evolution pattern of aging level, causes, and increasing rate from 2011 to 2015 in Henan province. The analysis data come from the Henan spatial database and Statistical Yearbook(2011-2015). 


\section{Geospatial-temporal characteristics of population aging and its evolution pattern in Henan province}

Geospatial-temporal characteristics of population aging and its evolution pattern. From 2011 to 2015, the province's aging levels (the proportion of population over 65 years in total population, same below) have been increasing, aging cities gradually increase. By the end of 2011 and 2012, the aging levels more than $7 \%$ have 15 cities, covering $97.56 \%$ of the province's area, the elderly populations respectively are 7.86 and 8.08 million, and the province's aging levels respectively are $8.36 \%$ and 8.61\%. By the end of 2013 and 2014, there are 16 cities with the aging levels greater than 7\%, covering $98.71 \%$ of the province's area, the elderly populations respectively are 8.30 and 8.53 million, the overall aging levels respectively are $8.82 \%$ and $9.06 \%$. By the end of 2015 , the province enter the aging society in general, the province's aging level is up to $9.37 \%$, more than $10 \%$ has a city, accounting for $70.92 \%$ of the province's area, the elderly populations are more than 8.84 million, Zhumadian is most serious regional with aging level $11.69 \%$.

In the view of geospatial distribution (Fig. 1), the aging levels in the southern part are greater than in northern regions. In 2011-2015, the area with high aging levels, as Zhumadian and Luohe, begin spreading to the surrounding, by the end of 2014, the cities as Zhoukou and Shangqiu in the southeast part and Xinyang in the south part have run into serious aging region, by the end of 2015, the development trend pace has accelerated, the serious aging region covering almost all cities in the southeast part, there are 10 cities possessing more than $10 \%$ aging level, 9 cities located in the southeast region, 1 located in north region. For the absolute amount of aging populations, 4 cities, as Nanyang, Zhoukou, Zhengzhou, Zhumadian, has more than 800 thousand elderly population as 1000, 94, 82 and 81 thousand respectively.

Table.1 The classification of population aging in 2015

\begin{tabular}{|c|c|c|c|c|c|c|c|c|c|c|c|c|c|c|c|c|c|c|}
\hline City & $\mathrm{XC}$ & $\mathrm{LH}$ & SQ & $\mathrm{XY}$ & $\mathrm{ZK}$ & ZMD & KF & LY & PDS & $\mathrm{AY}$ & $\mathrm{XX}$ & SMX & $\mathrm{NY}$ & $\mathrm{ZZ}$ & HB & $\mathrm{JZ}$ & PY & JY \\
\hline Aging $\mathrm{T}$ & \multicolumn{6}{|c|}{1} & & \\
\hline Aging center & \multicolumn{6}{|c|}{10.95} & \multicolumn{6}{|c|}{9.96} & \multicolumn{6}{|c|}{8.66} \\
\hline Birth Rate & $\mathrm{L}$ & M & $\mathrm{L}$ & $\mathrm{L}$ & $\mathrm{L}$ & $\mathrm{L}$ & $\mathrm{H}$ & $\mathrm{L}$ & $\mathrm{L}$ & $\mathrm{L}$ & $\mathrm{H}$ & $\mathrm{L}$ & $\mathrm{H}$ & M & $\mathrm{L}$ & $\mathrm{L}$ & $\mathrm{L}$ & $\mathrm{M}$ \\
\hline Death Rate & $\mathrm{M}$ & $\mathrm{L}$ & $\mathrm{M}$ & $\mathrm{M}$ & $\mathrm{M}$ & $\mathrm{M}$ & $\mathrm{H}$ & $\mathrm{M}$ & $\mathrm{M}$ & $\mathrm{M}$ & $\mathrm{H}$ & $\mathrm{M}$ & $\mathrm{H}$ & $\mathrm{L}$ & $\mathrm{M}$ & $\mathrm{M}$ & $\mathrm{M}$ & $\mathrm{L}$ \\
\hline Adult Export & $\mathrm{H}$ & $\mathrm{M}$ & $\mathrm{H}$ & $\mathrm{H}$ & $\mathrm{H}$ & $\mathrm{H}$ & $\mathrm{L}$ & $\mathrm{H}$ & $\mathrm{H}$ & $\mathrm{H}$ & $\mathrm{L}$ & $\mathrm{H}$ & $\mathrm{L}$ & $M$ & $\mathrm{H}$ & $\mathrm{H}$ & $\mathrm{H}$ & $\mathrm{M}$ \\
\hline
\end{tabular}
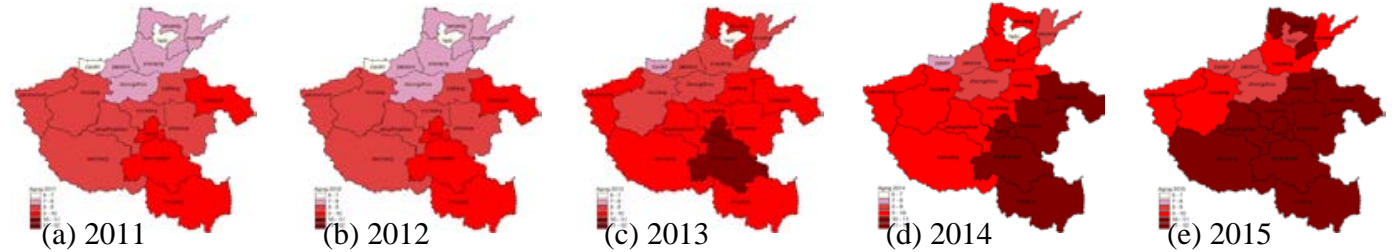

Fig.1 The geospatial distribution of population aging in each year

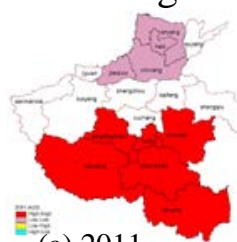

(a) 2011

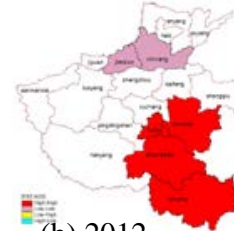

(b) 2012

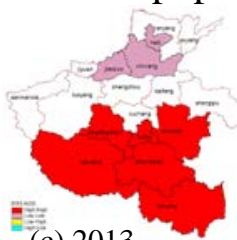

(c) 2013

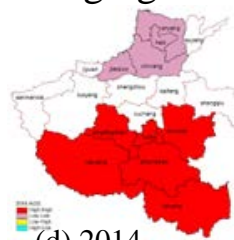

(d) 2014

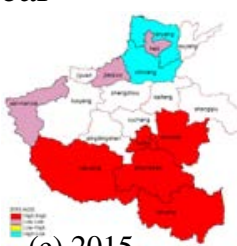

(e) 2015

Fig.2 The geospatial aggregation characteristics of population aging in each year

Geographically (Fig. 2), the spatial aggregation phenomenon of aging levels is very obvious (significance level greater than 5\%) in 2011-2015. In 2011-2013, there are obviously high-high spatial aggregation in the southeast region, and low-low spatial aggregation in the northern region. However from 2013, this aggregation phenomenon has been weakening, Moran I index and corresponding z-value gradually decrease (0.3748-0.3687-0.3562, 4.61-4.53-4.35). In 2015, some local high-low aggregation region appear in the region covering Xinxiang and Anyang. All of these mean the city aging levels in the province gradually tends to be balancedl [17-20]. 
main causes of population aging. According to aging causes, the population aging can be divided into 3 type as: a large number of young workers outflow resulting Spatial Residue Type, the living and health conditions improve, making the average life expectancy extended forming Everage Life Expectancy extended Type, Low Birth Rate Typ. The main causes of aging generally is the outflow of young workers in the economic developing regions, the low birth rate in the economic developed regions in the province $[1,3,4,6]$.

According the digital characteristics of aging levels, using K-mean clustering analysis method, under Euclidean distance and the sum of squares of deviations [17,18,20], the aging levels of 18 cities can be divided into 3 classes: serious aging cities (aging levels greater than 10\%), moderate aging cities (aging levels between 9\% and 10\%) and aging cities (aging levels less than $8 \%$ ). According the three main causes for population changes, the three classes can be divided into 6 subclasses as, serious aging cities with low birth rate and high young works exporting, serious aging cities with natural population change, moderate aging cities with low birth rate and high young works exporting, moderate aging cities with natural population change, aging cities with low birth rate and high young works exporting, aging cities with natural population change.

6 serious aging cities(Table 1), all located in the southeast region(Fig. 3 ), of which 5 cities belong to the low birth rate and high young works

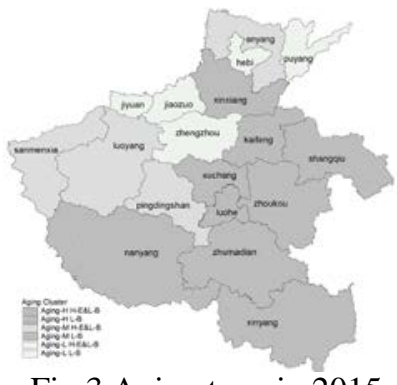

Fig.3 Aging type in 2015 exporting subclass, with high labor export and low natural population growth, respectively are Xuchang, Shangqiu, Xinyang, Zhoukou, Zhumadian, of which Luohe belongs to the natural population change subclass with average labor export and high natural population change. 7 moderate aging cities, of which 4 cities belong to the low birth rate and high young works exporting subclass as Luoyang, Pingdingshan, Anyang and Sanmenxia, of which 3 cities are the natural population change subclass with average labor export and high natural population change, as Kaifeng, Xinxiang and Nanyang. 5 aging cities, of which 3 cities belong to the low birth rate and high young works exporting subclass as Hebi, Jiaozuo and Puyang, of which 2 cities are the natural population change subclass with average labor export and high natural population change, as Zhengzhou and Jiyuan.

Combined with the city economic development data of corresponding year, it can be seen that cities in the southeast part, with developing economic and high population density, have high aging levels, exists significantly geospatial aggregation phenomenon, for the young works exporting and low natural birth rate, form the serious aging region in the province. Cities in the middle part along Longhai railway line, with relatively developing economic, have relatively low aging levels, does not exist any geospatial aggregation phenomenon, their main aging cause is natural population change.

Table.2 The population aging rate(2011-2015)

\begin{tabular}{|l|l|l|l|l|l|l|l|l|l|l|l|l|l|l|l|l|l|l|}
\hline & XC & PDS & HB & ZZ & KF & LY & AY & JZ & XX & PY & SMX & LH & ZMD & NY & JY & SQ & XY & ZK \\
\hline $11-12$ & 5.91 & 7.11 & 5.10 & 1.25 & 8.96 & 7.68 & 13.78 & 11.53 & 6.26 & 5.69 & 4.83 & 2.53 & 7.55 & 7.36 & 0.58 & 10.14 & 4.84 & 9.21 \\
\hline $12-13$ & 0.95 & 2.21 & 2.52 & 3.50 & 3.83 & 3.92 & 4.55 & 4.55 & 5.92 & 6.12 & 7.71 & 7.84 & 7.94 & 8.27 & 8.49 & 9.79 & 10.29 & 12.73 \\
\hline $13-14$ & 4.35 & 1.88 & 4.51 & 3.09 & 0.35 & 5.25 & 3.65 & -1.06 & 3.93 & 8.28 & 10.71 & 8.40 & 7.99 & 1.10 & 3.23 & 3.94 & 7.73 & 11.15 \\
\hline $14-15$ & 6.33 & 1.01 & 15.94 & -2.21 & 7.81 & 5.49 & 4.79 & 6.69 & 5.69 & -1.63 & -2.58 & 2.11 & 5.03 & 3.86 & 15.77 & 3.51 & 4.82 & 6.47 \\
\hline
\end{tabular}

\section{Geospatial-temporal characteristics of aging rate and its evolution pattern in Henan province}

Geospatial-temporal characteristics of aging rate and its evolution pattern. From 2011 to 2015, along with the province's aging level continues increasing, all cities enter the aging society. By the end of 2012, the aging levels of Jiyuan and Hebi remain below 7\%. By the end of 2012, Jiyuan firstly enter the aging society, by the end of 2012, the aging level of Hebi has reached the level of $8.01 \%$.

2011 to 2012 (Table. 2), the aging rates of 13 cities are more than 5\% (Fig. 4), of which the northern cities as Jiaozuo and Anyang, eastern city as Shangqiu have more than $10 \%$ of aging levels. In the spatial distribution, the aging rates in the northern part are faster than the southern part. 
From 2012 to 2013, compared to the last period, the aging rate has accelerated from $2.28 \%$ to $2.45 \%$, the aging rate of Xuchang is the lowest in the province, less than $1 \%$. There are 10 cities greater than 5\%, of which Zhoukou in the eastern part and Xinyang in the southern are more than $10 \%$. The pattern of the spatial distribution is on the contrary to the last period, the aging rates in the southern part are faster than the northern part.

From 2013 to 2014, the aging rate reaches up to $3.42 \%$, Jiaozuo is only one city the aging rate is negative as $-1.06 \%$. There are 7 cities , the aging rates are greater more than $5 \%$, of which Zhoukou in the western part and Sanmenxia in the western part reach more than $10 \%$. In the spatial

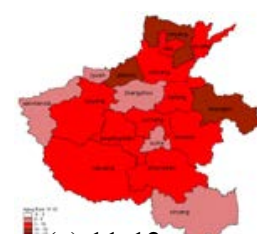

(a) $11-12$

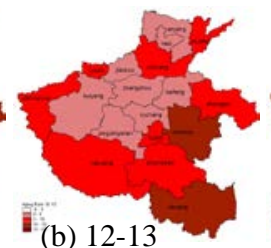

(b) $12-13$

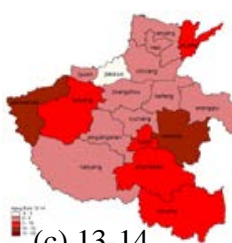

(c) $13-14$

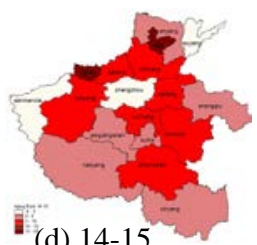

(d) $14-15$

Fig.4 The geospatial distribution of aging rate in each year distribution, the aging rates in the southern part are faster than the northern part.

From 2014 to 2015, the aging rate has weakened running to $2.81 \%$, but still higher than the first two periods, there are 3 cities as Sanmenxia, Zhengzhou and and Puyang with negative aging rates, respectively are $-2.59 \%,-2.21$ and $-1.63 \%$. There are 9 cities with the aging rates more than $5 \%$, of which Hebi and Jiyuan respectively reach to $15.77 \%$ and $15.94 \%$, which are the only once the aging rates more than $15 \%$ in the four periods.

Overall, in the four periods, the aging rates have gradually been increasing, the difference between the cities have been enlarging.Geospatial correlation analysis of aging rate. According the digital characteristics of ageing rates, using K-mean clustering analysis on the four periods, the 18 cities presents clearly (the distance within classes

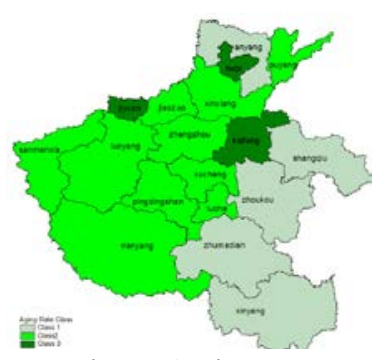

Fig.5 Aging rate are far less than the distance between classes) 3 classes(Fig.5). The first class includes Anyang, Zhumadian, Shangqiu, Xinyang and Zhoukou, the aging levels in each period maintain rapid growth rates, the absolute rates gradually decrease, the acceleration gradually increases. The second class includes Xuchang, Pingdingshan , Zhengzhou, Luoyang, Jiaozuo, Xinxiang Puyang, Sanmenxia, Luohe and Nanyang, the aging levels in each period keep average growth rate, the absolute rates gradually decrease, the acceleration is much less than the first class. The third class includes Hebi, Kaifeng and Jiyuan, the aging rates in the first three periods is very small, but the acceleration in the forth period is very large. Geographically, first class mainly is distributed in the southeast part, the second class is distributed in the middle and southwestern part, the third class is distributed in northern part of the province.

In the 4 periods, the geospatial aggregation of aging rates is significantly less than that of the aging level (Fig. 6). In the first period, there are a high-high aggregation region around Puyang, and a high-low

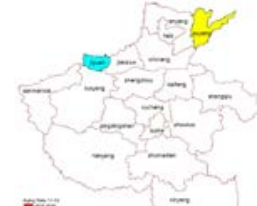

(a) $11-12$

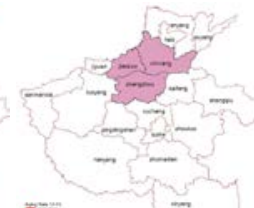

(b) $12-13$

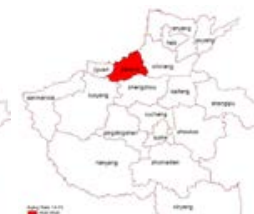

(c) $13-14$

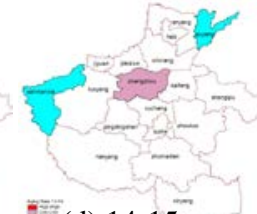

(d) $14-15$

Fig.6 The geospatial aggregation characteristics of aging aggregation region around Jiyuan. In the second period, Zhengzhou Xinxiang and Jiaozuo compose of low-low aggregation region. In the third period, Jiaozuo has the characteristics of high-high aggregation. In the fourth period, Zhengzhou is high-high aggregation region, Puyang and Sanmenxia show high-low aggregation. In other words, the geospatial distribution of aging rates is relatively balanced, the aging rates in central region is lower than the border region in the province.

\section{Conclusion}

Based on the quantitative analysis of the aging data in 2011-2015, the geospatial-temporal characteristics and evolution pattern of aging level, causes, and increasing rate are studied, and get the following results 
i) The distribution of population aging levels has significant regional differences in the province, the aging levels in southeastern part are significantly higher than other regions, The regions with low aging levels have relatively developed economics, the regions with high aging levels have developing economics high population density.

ii)The main causes of population aging is residual space type and low birth rate type. The serious aging regions have relatively high young works exporting and low birth rate it results. The weak aging regions have some young works importing and relatively high birth rate it.

iii)The aging rates have gradually been increasing, the difference between the cities have been enlarging, aging level and the aging rate has some negative correlation.

iv)The geospatial aggregation of aging levels is significant, southeast part is the high-high aggregation region and northern part is low-low aggregation region, but the aggregation characteristics are becoming weak. The aggregation characteristics of aging rate is relatively weak, there are some weak low-low aggregation characteristics around Zhengzhou, provincial capital, the geospatial distribution of aging rates is relatively balanced, the aging rates in central regions are lower than the border regions.

\section{References}

[1] Haitao Yu, Apatial-temporal features and influential factors of China urban floating population growth. Chinese Journal of Population Science, 2012, 4, p.47-58.

[2] Haitao Yu, Urban growth in China. Journal of Urban and Regional Planning, 2012, 2, p.62-79.

[3] Lei Sun, Tianjiao Chang, Quanliu Guo, On the spatial distribution of population aging and its synchronization with economic development in China. Journal of East China Normal University (Humanities and Social Sciences, 2014, 3, p.123-132.

[4] Zeyu Wang, Research on the measure of Chinese population aging level and spatial correlation. Areal Research and Development, 2013, 3, p.138-143.

[5] Huajun Liu, Liwei Li, Sai Yang, Spatial inequality and distributional dynamics of population aging in China, 1989-2011. Population Research, 2014, 38(2), p.71-82.

[6] Zhang X, Zhu X Y, Bao S M, Spatial-temporal pattern analysis and spatial disparity research on provincial total fertility rate in China. Geomatics and Information Science of Wuhan University, 2012, 37(5), p. 516-519.

[7] Zhao R Y, Liu C, Zhang F, Spatial econometric research on regional spillover and distribution difference of population aging in China. Population Research, 2012, 36(2),p.71-81.

[8] Weihua Yi, Xinyue Ye, Zheye Wang, The spatial aging pattern and its driving forces in Guangdong. Population \& Economics, 2015, 3,p.33-42.

[9] Kaizhou Zhang, Nan Chen, Characteristics of spatial-temporal evolution in population aging and driving mechanism at county level in Fujian Province during 1990-2010. Progress in Geography, 2014, 32(5), p.605-612.

[10] Zhang Z B, Pan J, Da F W, Population spatial structure evolution pattern and regulating pathway in Lanzhou city. Geographical Research, 2012, 31(11), p. 2055-2068.

[11] Chunshan Zhou, Yixuan Li, Xinmei Tong, Spatial change and influence factor of population aging in Guanzhou during 2000-2010. Acta Scientiarum Naturalium Universitatis Sunyatseni, 2016, 55(1), p.114-122.

[12] Yinhong Lu, The Spatial distribution of aging population and its conformity with living environment in Shanghai. Modern Urban Research, 2013, 10, p.94-98. 
[13] Po Xie, Jie Zhou, A research on spatial patterns and development trajectory of the elderly population in the metropolis taking Beijing, Shanghai, Guangzhou and Wuhan as examples. Urban Planning Forum, 2013, 210(5),p.56-62.

[14] Chengdong Yi, Chun Zhang, Shuping Wu, Spatial restructuring of fenior population in Beijing from 2000-2010. Urban development studies, 2014, 21(2), p.66-70.

[15] Yajie Zhang, Liangqian Ye, Qingjun Shao, Temporal-spatial difference of population aging in China. Geomatics \& Spatial Information Technology, 2015, 38(12),p.18-26.

[16] Xiangqiu Huang, Changhong Miao, Yongjian Ma, Spatial pattern of the floating population and its influence on population distribution in Henan province. Areal Research and Development, 2014, 33(4).p.142-147.

[17] Pengxu Tian, Yong-xin Zhou, Fang He, The evolution of the urban residential space under the aging trend based on hierarchical clustering analysis, Journal of Shandong Agricultural University (Natural Science Edition ), 2015, 46(3), p.470-475.

[18] Bingyun Zheng, The clustering analysis of multivariable pnael data and its application. Application of Statistics and Management, 2008, 27(2),p.265-268.

[19] Jinliang Wang, Zhilin Huang,Jingan Shao, Gradient distribution of belt transects and characteristics of spatial clustering of point pattern of woodland landscapes: A case study of ecological barrier zone of Three-Gorges Reservoir in Chongqing. Progress in Geography, 2013, 32(2), p.308-317.

[20] Zhao Y, Wang Y S, Progress of spatial analysis. Geography and Geo-Information Science, 2011, 27(5), p. 2-8. 